\title{
Rotational Temperature Behaviour in Supersonic Jet Expansions of Molecular Nitrogen
}

\author{
A. A. Ilyukhin ${ }^{1}$, R. L. Pykhov ${ }^{1}$, V. V. Smirnov ${ }^{1}$, and G. Marowsky ${ }^{2}$ \\ 1 Institute of General Physics of the Academy of Sciences of the USSR, 38 Vavilova Street, \\ SU-117942 Moscow, USSR \\ 2 Abteilung Laserphysik, Max-Planck-Institut für Biophysikalische Chemie, Am Fassberg, D-3400 Göttingen, \\ Fed. Rep. Germany
}

Received 22 December 1989/Accepted 18 April 1990

\begin{abstract}
The rotational temperature behaviour in adiabatic jet expansion of molecular nitrogen is investigated over a wide range of stagnation density and nozzle diameter values using coherent anti-Stokes Raman spectroscopy (CARS). Estimates of rotational collisional numbers are made for both $\left(\mathrm{N}_{2}+\mathrm{N}_{2}\right)$ and $\left(\mathrm{N}_{2}+\mathrm{Ar}\right)$ systems. The aspect of cluster formation for both systems is also discussed.
\end{abstract}

PACS: 42.65

Supersonic free-expansion jets can be effectively used for solving a number of problems connected with lowtemperature and low-pressure spectroscopy, translational, rotational, and vibrational relaxation processes, cluster structure and condensation dynamics [1-5]. Detailed information (on the molecular level) about the processes in the flow can be obtained by several spectroscopic techniques, such as electron-beam diagnostics, laser-induced fluorescence, infrared absorption, and Raman scattering [6-11]. During recent years a number of coherent nonlinear scattering techniques for diagnostics of Raman-active transitions have been successfully used for temperature and density measurements [12-17], and for investigation of condensation processes in supersonic jets [18-20].

In this paper we report the results of an investigation of rotational temperature behaviour in adiabatic jet expansion of molecular nitrogen over a wide range of stagnation density and nozzle diameter values using coherent anti-Stokes Raman spectroscopy (CARS). Estimates of rotational collisional numbers are made for both $\left(\mathrm{N}_{2}+\mathrm{N}_{2}\right)$ and $\left(\mathrm{N}_{2}+\mathrm{Ar}\right)$ systems. The aspect of cluster formation for both systems is also discussed.

\section{Experimental}

The experimental details, advantages and disadvantages of the CARS technique for many applications can be found in [13], so here we discuss only the most important features of the installation.

The pulsed CARS spectrometer incorporates a single-frequency $\mathrm{Nd}$ :YAG laser with two amplifiers delivering $30 \mathrm{~mJ}$ at $1064 \mathrm{~nm}$ with $0.005 \mathrm{~cm}^{-1}$ frequency bandwidth (FWHM) and $10 \mathrm{~Hz}$ repetition rate. Its output is frequency doubled and the $532 \mathrm{~nm}$ beam ( $20 \%$ of the total energy), separated by a polarization filter, is used to pump a tunable dye laser. The remaining $1064 \mathrm{~nm}$ beam is frequency-doubled to produce the pump beam for the CARS process. The dye laser is operated with Phenalemin-160 and provides the Stokes beam within a continuous tuning region of $600 \mathrm{~cm}^{-1}$ centered at $607 \mathrm{~nm}$. Typical laser pulse energies are $7 \mathrm{~mJ}$ in the pump beam and $0.1 \mathrm{~mJ}$ in the Stokes beam. The spectral resolution of the spectrometer is limited by the dye laser bandwidth and is equal to $0.05 \mathrm{~cm}^{-1}$.

Two nozzle types are employed to obtain the freeexpansion jet: continuously operating thin-walled pinhole orifices with diameters $d=37 \mu \mathrm{m}$ or $d=95 \mu \mathrm{m}$, and a pulsed valve of $510 \mu \mathrm{m}$ diameter with a sonic nozzle producing gas pulses of approximately $1 \mathrm{~ms}$ duration. The nozzle assembly is mounted on a three-dimensional translation stage and can be positioned relative to the fixed laser beams.

The stagnation number density was in the range $n_{0}=2.41 \times 10^{25}-1.45 \times 10^{26} \mathrm{~m}^{-3} \quad$ (corresponding to stagnation pressures of approximately 1-6 bar), and the background pressure changed accordingly from 35 to $100 \mathrm{~Pa}$.

Both pure nitrogen and a nitrogen-argon mixture with pressure ratio $1: 10$ were in the experiments.

The three-dimensional BOXCARS [21] interaction of laser beams is used to increase the spatial resolution of the spectrometer and to eliminate the contribution to the signal of the atmospheric nitrogen. The pump beam at 

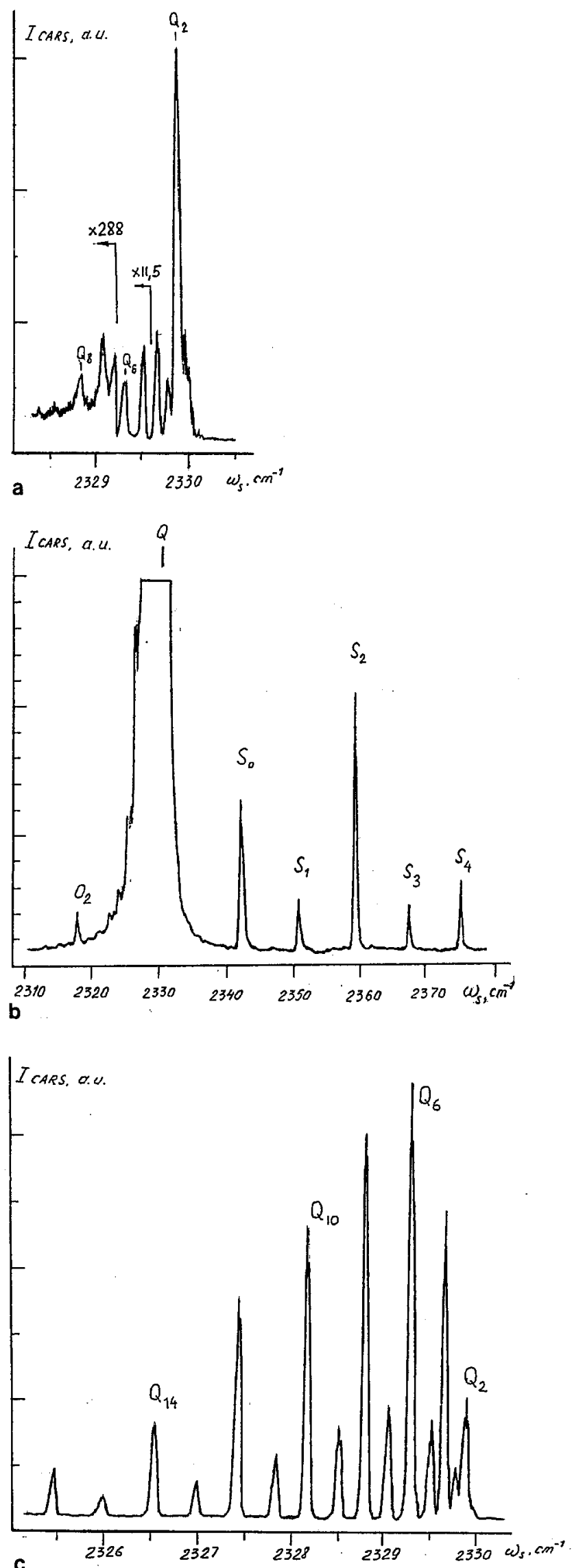

Fig. 1a-c. Typical CARS spectra of nitrogen: a $Q$-branch, supersonic jet, rotational temperature $T_{\mathrm{r}}=35 \mathrm{~K} ; \mathbf{b} S$-branch, same conditions; c $Q$-branch, static conditions, $T=300 \mathrm{~K}, P=60$ Torr
$532 \mathrm{~nm}$ is split into two beams with equal intensity, which are parallel to and spatially separated from the Stokes beam. The three beams are focused with a $80 \mathrm{~mm}$ focal length lens into the expansion jet at relative angles of about $7^{\circ}$. The measured spatial resolution of the spectrometer is $30 \times 30 \times 400 \mu \mathrm{m}$. The CARS signal, after separation from the pump beams by a fourprism filter, is detected by a photomultiplier, averaged with a boxcar integrator, and then plotted on a chart recorder.

\section{Results and Discussion}

Resolved CARS spectra of the $Q$-branch $(\Delta v=1, \Delta J=0)$ transition in the electronic ground state of nitrogen were recorded on the axis of the supersonic jet at different normalized distances $x / d$ from the nozzle orifice. At small distances $S$-branch $(\Delta v=1, \Delta J=2)$ CARS spectra were also recorded. Examples of nitrogen CARS spectra for $Q$ and $S$-branch spectra in a supersonic jet indicating a rotational temperature $T_{\mathrm{r}}=35 \mathrm{~K}$ are presented in Fig. $1 \mathrm{a}$ and $\mathrm{b}$, respectively. Figure 1c shows for comparison the CARS $Q$-branch spectra of nitrogen recorded under static room-temperature conditions. Information about the rotational distribution functions (RDFs) was in most cases derived from the peak intensities of the CARS spectra. The extracted distribution functions turned out to be nearly Boltzmann and allowed one to determine the local values of the rotational temperature along the flow axis. At large distances from the nozzle orifice the recorded spectra contained only the partially resolved lines with rotational quantum numbers $J=0, J=1$, and $J=2$. In these cases the rotational temperature was determined from fitting the theoretically calculated CARS spectra to the experimental data.

The main features of kinetic processes in the flow depend on the value of the local Knudsen number $K_{n}[5]$ :

$K_{n}=C\left(\frac{x}{d}\right)^{\gamma} \frac{1}{T_{0}} \frac{1}{\left(n_{0} d\right)}$,

where $\gamma=c_{\mathrm{p}} / c_{\mathrm{v}}$ is the ratio of specific heats and $C$ is a gasdependent constant. If the stagnation temperature $T_{0}$ is kept constant, changing the nozzle diameter $d$ (leading to a change of the time scale of gas expansion) or the stagnation density $n_{0}$ (and thus the frequency of molecular collisions) can significantly affect the dynamics of the internal degrees of freedom of molecules in the flow. Examples of the dependence of the measured rotational temperature on the normalized distance $x / d$ in the flow for different values of the parameter $n_{0} d$ are presented in Fig. 2 both for the $\mathrm{N}_{2}+$ Ar mixture $(a, b, c)$ and for pure nitrogen $(d, e, f)$. Good agreement between the measured rotational temperatures and the theoretically calculated isentropic temperatures [22] is observed in the $\mathrm{N}_{2}+\mathrm{Ar}$ mixture for $n_{0} d=1.17 \times 10^{22} \mathrm{~m}^{-2}$ up to $x / d \sim 5$ (Fig. 2b). In this case the rotational and translational degrees of freedom are in equilibrium, and direct determination of gas temperature from the rotational distribution functions is possible. Similarly, equilibrium flow in pure nitrogen takes place for $n_{0} d=2.5 \times 10^{22} \mathrm{~m}^{-2}$ (Fig. 2e). 

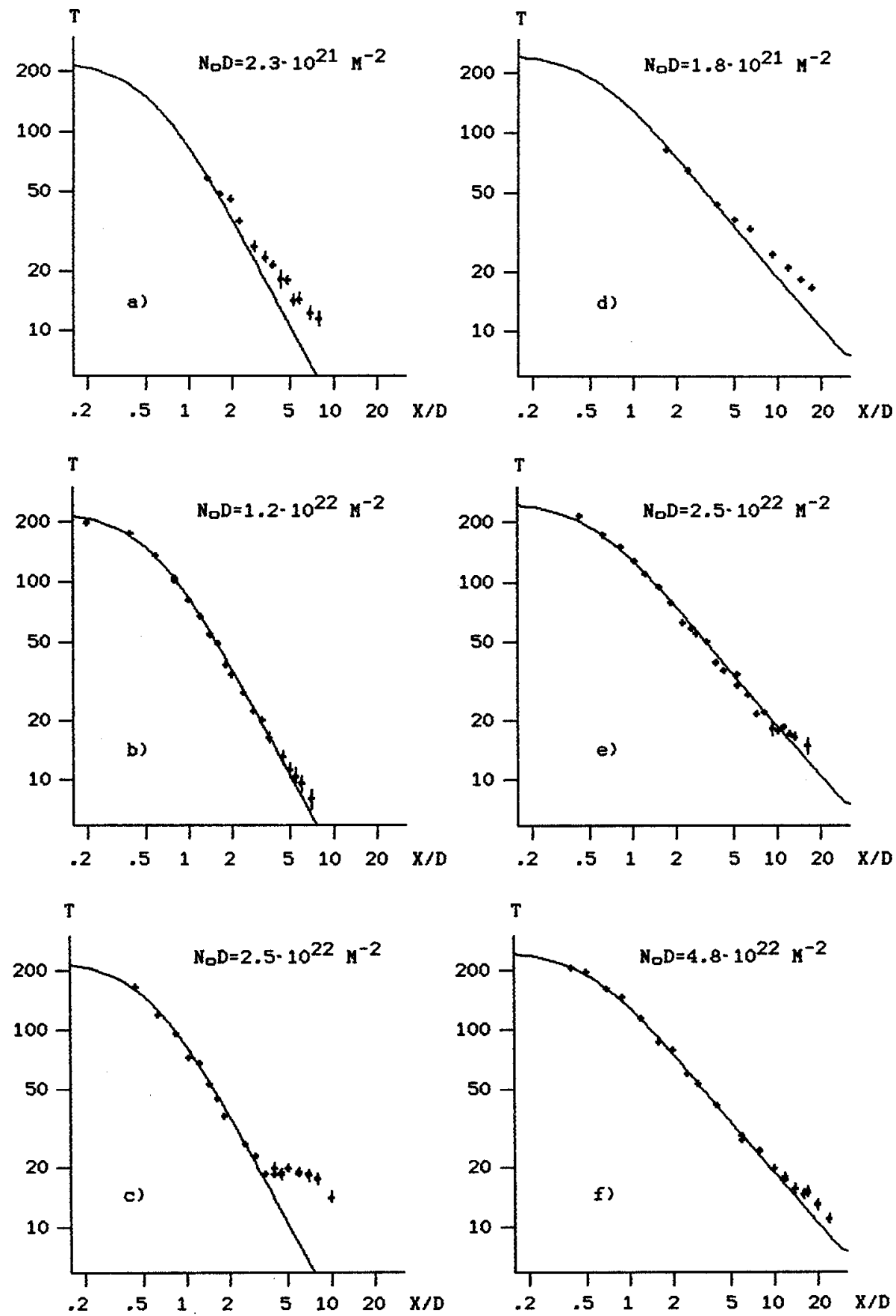

Fig. 2a-f. Rotational temperature as a function of normalized distance from the nozzle orifice: a-c the $\mathrm{N}_{2}+\mathrm{Ar}$ mixture $1: 10 ; \mathbf{d}-\mathrm{f}$ pure $\mathrm{N}_{2}$. The nozzle diameter $d$ is a $95 \mu \mathrm{m}, \mathbf{d} 37 \mu \mathrm{m}, \mathbf{b}-\mathbf{c}, \mathbf{e}, \mathbf{f}$ $510 \mu \mathrm{m}$; stagnation temperature $T_{0}=300 \mathrm{~K}$. Solid line represents isentropic temperature calculations

At lower densities (and hence lower collision numbers) or smaller nozzle diameters (faster speeds of gas parameter changes in the flow) the behaviour of rotational temperatures is governed by the processes of rotational relaxation. In this $n_{0} d$ range we observe a flow type with non-equilibrium between rotational and translational degrees of freedom. A slow deviation of the measured rotational temperature values from the calculated isentropic translational temperature takes place, starting from $x / d=1.8$ for $\mathrm{N}_{2}+\operatorname{Ar}$ (Fig. 2a) and $x / d=5$ for pure $\mathrm{N}_{2}$ (Fig. 2d). Since in our experiments we have not observed deviations of the distribution functions from Boltzmann type (within the available range of rotational states accessible in our CARS experiments), estimation of the rate constants for level-to-level rotational transitions was not possible. Still, our measurements of rotational temperature behaviour allow us to estimate the effective rotational collision number

$Z_{\mathrm{r}}=\tau_{\mathrm{rt}} / \tau_{\mathrm{t}}=\tau_{\mathrm{rt}}^{4} n \sigma_{\mathrm{t}}^{2} \sqrt{\pi k T_{\mathrm{t}} / m}$

from the equation for energy balance [3]:

$\frac{d T_{\mathrm{r}}}{d t}=-\frac{1}{\tau_{\mathrm{rt}}}\left(T_{\mathrm{r}}-T_{\mathrm{t}}\right)$,

where $\tau_{\mathrm{rt}}$ is the rotational relaxation time, $\tau_{\mathrm{t}}$ the mean time between collisions, $\sigma_{\mathrm{t}}=\sigma_{0}\left(1+C / T_{\mathrm{t}}\right)$ a gas-kinetic crosssection, $m$ the reduced mass, $k$ the Boltzmann constant, $T_{t}$ the gas temperature, $T_{\mathrm{r}}$ the rotational temperature, and $n$ the number density. The gas-kinetic cross-section con- 


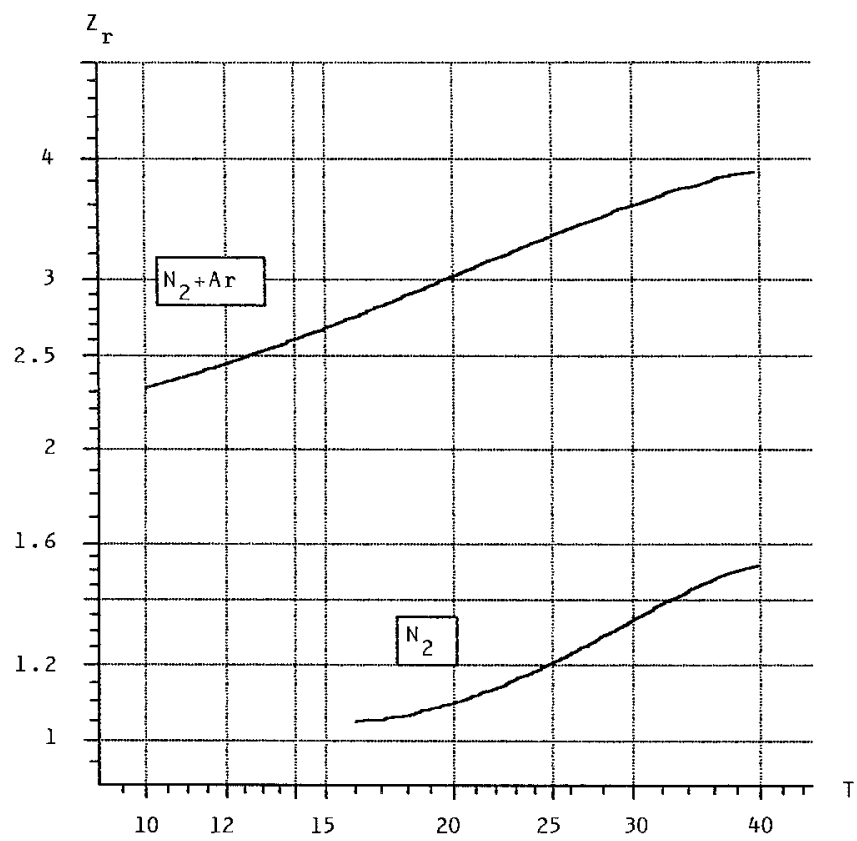

Fig. 3. Rotational collision number $Z_{\mathrm{r}}$ as a function of temperature for pure $\mathrm{N}_{2}$ and for the $\mathrm{N}_{2}+$ Ar mixture. For details see text

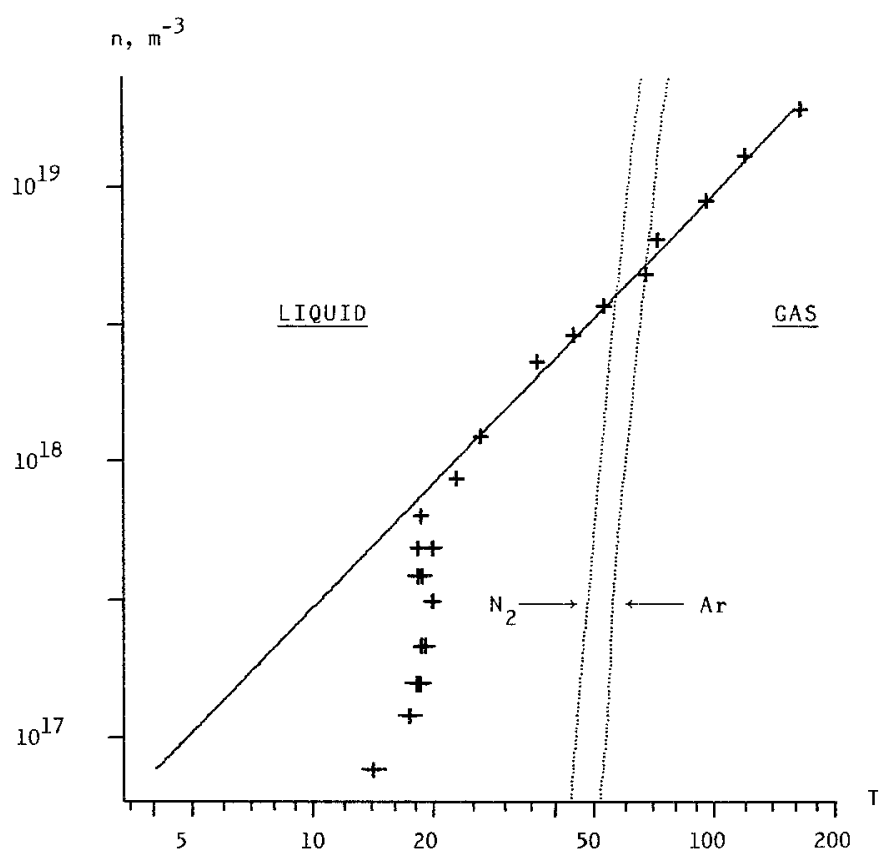

Fig. 4. Temperature $T$ vs number density $n$ for the $\mathrm{N}_{2}+$ Ar mixture, $T_{0}=300 \mathrm{~K}, n_{0} d=2.46 \times 10^{22} \mathrm{~m}^{-2}$. Crosses represent the measured values of rotational temperature; the solid line gives the isentropic temperature calculations. Dotted lines separate the areas of existence of liquid and gaseous phases, according to [25]

In the case of pure nitrogen there is also a deviation from the isentropic temperature for $n_{0} d>3 \times 10^{22} \mathrm{~m}^{-2}$ starting at $x / d \sim 10$ (Fig. $2 \mathrm{f}$ ). In this case, however, we have observed a significant decrease in intensity for CARS spectral lines corresponding to the rotational levels $J=0$ and $J=1$. To check this phenomenon further we have used a conical supersonic nozzle with throat diameter $350 \mu \mathrm{m}$ and cone angle $31^{\circ}$, which is approximately equivalent to a sonic nozzle with a diameter of $1.077 \mathrm{~mm}$ [5]. This allowed us to increase the gas density in the flow and to record additionally the $S$-branch CARS spectra of nitrogen. In both $Q$ - and $S$-branch spectra we have observed a decrease in intensity and an anomalous broadening of the lines corresponding to rotational levels $J=0$ and $J=1$. The distribution functions extracted from the peak CARS intensities show the underpopulation of these states (Fig. 5). These results allow the assumption that at the primary stage of condensation clusters are formed from those molecules occupying the lowest rotational states for different spin modifications. A similar effect was observed earlier for the condensing flow of heavy water molecules [24]. However, we must mention that the anomalously strong broadening of the CARS spectral lines corresponding to $J=0$ and $J=1$ levels can lead to a decrease of the peak intensities of the CARS signal and thus to a decrease of the extracted populations of these levels. Final conclusions on this phenomena can only be drawn after additional high-resolution investigations of collisionally broadened CARS spectra at low temperatures.

Figure 6 summarizes the dependence of the measured rotational temperature $T_{\mathrm{r}}$ on the parameter $n_{0} d$ at fixed distance $x / d$ for $\mathrm{N}_{2}+\operatorname{Ar}$ mixtures. The minimum value of $T_{\mathrm{r}}$ for $n_{\mathrm{o}} d=1.17 \times 10^{22} \mathrm{~m}^{-2}$ corresponds to the equilib- 


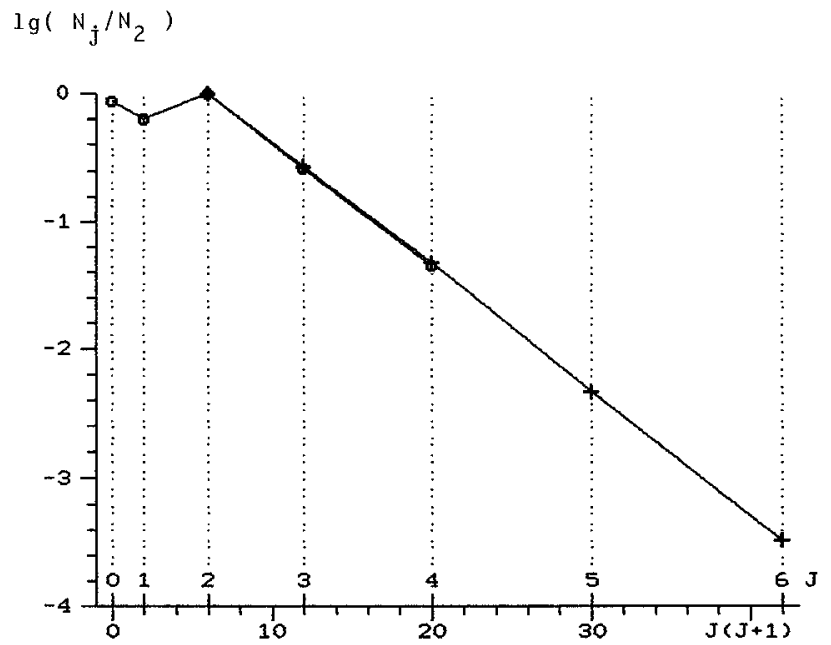

Fig. 5. Rotational distribution function of nitrogen in a supersonic jet expansion from the conical nozzle. $(+) Q$-branch measurements; $(O)$ $S$-branch measurements

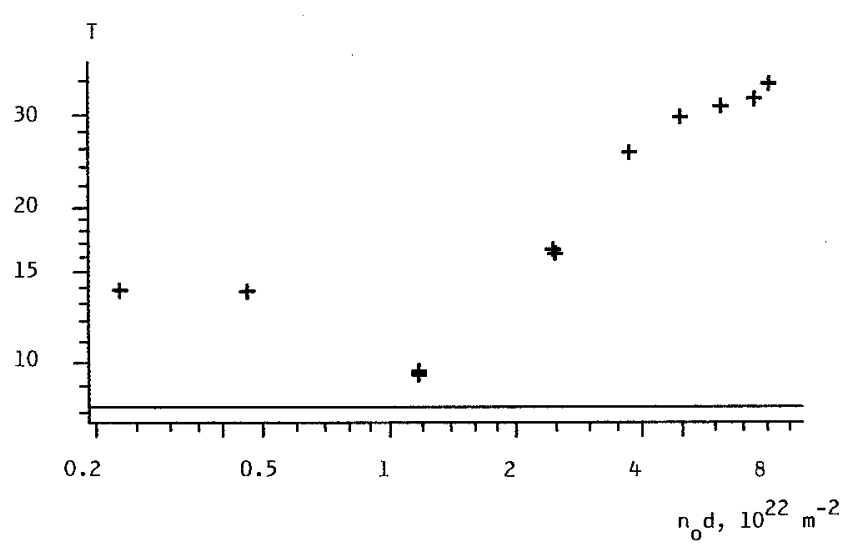

Fig. 6. Rotational temperature as a function of the parameter $n_{0} d$ at fixed distance $x / d=6$ for the $\mathrm{N}_{2}+\mathrm{Ar}$ mixture. The straight line at $T=8.2 \mathrm{~K}$ represents the calculated isentropic temperature

rium between the rotational and translational degrees of freedom in the flow. The increase of $T_{\mathrm{r}}$ at lower values of $n_{0} d$ is explained by the fact that the rotational relaxation processes are too slow to follow the expansion law, and a part of the initial rotational energy remains "frozen" in the rotational degrees of freedom. The increase of $T_{\mathrm{r}}$ at higher values of $n_{0} d$ is due to the release of energy from the condensation process into the flow.

\section{Conclusions}

Our results demonstrate that coherent anti-Stokes Raman spectroscopy is an effective and universal tool for local non-perturbing diagnostics of gas parameters in supersonic jets. It allows quantitative information to be obtained about the distribution of energy between the internal degrees of freedom as well as about the characteristic times of relaxation processes in the flow. In addition, the CARS technique is particularly useful for studies of cluster formation, since, provided that the energy of the pump beams is kept sufficiently low, CARS probing has no effect on the condensation processes.

\section{References}

1. H. Ashkenas, F.S. Sherman: "The Structure and Utilization of Supersonic Free Jets in Low Density Wind Tunnels", in 4th Int. Symp. on Rarefied Gas Dynamics, Proc., Vol. 2, ed. by J.H. de Leeuw (Academic, New York 1966) pp. 84-105

2. D.H. Levy, L. Wharton, R.E. Smalley: "Laser Spectroscopy in Supersonic Jets", in Chemical and Biochemical Applications of Lasers, Vol. 2, ed. by C.B. Moore (Academic, New York 1977) pp. 1-41

3. R.J. Gallagher, J.B. Fenn: J. Chem. Phys. 60, 3487 (1974)

4. N.V. Karelov, A.K. Rebrov, R.G. Sharafutdinov: "Population of Rotational Levels of Nitrogen Molecules at Nonequilibrium Condensation in a Free Jet", in 11th Int. Symp. on Rarefied Gas Dynamics, Proc., Vol. 2 (Comissariat à l'énergie atomique, Paris 1979) pp. 1131-1140

5. O.F. Hagena: Surf. Sci. 106, 101 (1981)

6. E.P. Muntz: Phys. Fluids 5, 80 (1962)

7. B.N. Borzenko, N.V. Karelov, A.K. Rebrov, R.G. Sharafutdinov: J. Appl. Mech. Tech. Phys. 17, 615 (1976)

8. D. Bassi, A. Boschetti, S. Marchetti, G. Scoles, M. Zen: J. Chem. Phys. 74, 2221 (1981)

9. D.N. Travis, J.C. McGurk, D. McKeown, R.G. Denning: Chem. Phys. Lett. 45, 287 (1977)

10. H.P. Godfried, I.F. Silvera: Phys. Rev. A 27, 3008 (1983)

11. G. Luijks, S. Stolte, J. Reuss: J. Chem. Phys. 62, 217 (1981)

12. M.N. Osin, P.P. Pashinin, V.V. Smirnov, V.I. Fabelinskii, N.S. Tskhai: Sov. Tech. Phys. Lett. 6, 64 (1980)

13. S.I. Valyanskiy, K.A. Vereshchagin, A.Yu. Volkov, A.A Ilyukhin, P.P. Pashinin, V.V. Smirnov, V.I. Fabelinskiy: "Local Noninvasive Diagnostics of Gaseous Media", in Laser-Induced Raman Spectroscopy in Crystals and Gases, ed. by P.P. Pashinin, Proc. of the Institute of General Physics of the Academy of Sciences of the USSR, Vol. 2 (Nova Science, Commack, NY 1988) pp. $165-190$

14. P. Huber-Walchli, D.M. Guthals, J.W. Nibler: Chem. Phys. Lett. 67, 233 (1979)

15. J.J. Valentini, P. Esherick, A. Owyoung: Chem. Phys. Lett. 75, $590(1980)$

16. M.D. Duncan, P. Oesterlin, R.L. Byer: Opt. Lett. 6, 90 (1981)

17. S.A. Achmanov, N.I. Korotev, S.A. Magnitskii, V.B. Morosov, A.P. Tarasevich, V.G. Tunkin: J. Opt. Soc. Am. B 2, 640 (1985)

18. F. Konig, P. Oesterlin, R.L. Byer: Chem. Phys. Lett. 88, 477 (1982)

19. R. Beck, J.W. Nibler: Chem. Phys. Lett. 148, 271 (1988)

20. H.D. Barth, C. Jackschath, T. Pertsch, F. Huisken: Appl. Phys. B 45, 205 (1988)

21. A.C. Eckbreth: Appl. Phys. Lett. 32, 421 (1978)

22. P.A. Skovorodko, R.G. Sharafutdinov: J. Appl. Mech. Tech. Phys. 22, 629 (1981)

23. A.E. Belikov, A.I. Burshtein, S.V. Dolgushev, A.V. Storozhev, M.L. Strekalov, G.I. Sukhinin, R.G. Sharafutdinov: "Rate Constants and Times of Rotational Relaxation of Nitrogen in Argon"; Preprint of the Institute of Thermal Physics of Siberian Department of the Academy of Sciences of the USSR, Novosibirsk (1988) No. 182-88, 183-88

24. V.K. Konyukhov, A.M. Prokhorov, V.I. Tikhonov, V.M. Fajzulaev: Effect of Rotational Selectivity in Heterogenous Condensation of Heavy Water Vapours", in Physical Processes in Low-Temperature Gas-Dynamical Lasers, ed. by V.K. Konyukhov, Proc. of the Institute of General Physics of the Academy of Sciences of the USSR, Vol. 12 (Nauka, Moscow 1988) pp. $100-110$

25. D.E. Gray (ed.): American Institute of Physics Handbook (McGraw-Hill, New York 1972) Chap. 4k 